\title{
Benefícios na utilização da frota elétrica para ventilação de mina subterrânea
}

Leandro de Vilhena Costa ${ }^{1 *}$ (D)

José Margarida da Silva ${ }^{1}$

\section{Resumo}

O desenvolvimento de equipamentos elétricos permitiu a utilização de uma energia mais limpa em minas subterrâneas. A recente aplicação dessa tecnologia em carregadeiras LHD e caminhões viabilizou sua aplicação em minas no Canadá e na Suécia. Além de eliminar as emissões de poluentes, estima-se que a utilização da frota elétrica apresente um potencial de redução de aproximadamente $40 \%$ na demanda por ventilação devido a zero emissão de gases e da menor emissão de calor dos veículos elétricos. Entretanto, há poucos estudos técnicos e de aplicação na prática. Apesar dos benefícios ambientais, financeiros e de saúde ocupacional, observa-se a preferência pela tecnologia a diesel dificultando a expansão da frota elétrica em todo mundo. Nesse contexto, este artigo busca analisar para uma mina de ouro, localizada na região central de Minas Gerais, aplicação da tecnologia elétrica atualmente disponível para mineração e quantificar qual o potencial impacto sobre sistemas de ventilação com a substituição de $100 \%$ da frota diesel pela elétrica. Outra questão seria avaliar o potencial impacto que a adoção de frota elétrica representaria em uma mina nas condições brasileiras, respeitando as normas regulatórias vigentes.

Palavras-chave: Ventilação de mina; Equipamentos elétricos; Diesel; Emissão de calor.

\section{Benefits of using the electric fleet for underground mine ventilation}

\begin{abstract}
The development of electrical equipment allowed the use of more clean energy in underground mines. The recent application of this technology in LHD loaders and trucks has enabled its application in mines in Canada and Sweden. In addition to eliminating pollutant emissions, it is estimated that the use of the electric fleet has a potential reduction of approximately $40 \%$ in demand for ventilation due to zero emissions of gases and lower heat emissions from electric fleet. However, there are few technical and application studies in practice. Despite the environmental, financial and occupational health benefits, the preference for diesel technology is observed, making it difficult to expand the electric fleet worldwide. In this context, this article seeks to analyze the application of electrical technology currently available for mining and quantify the potential impact on ventilation systems with the replacement of $100 \%$ diesel by electric. Another issue would be to evaluate the potential impact that the adoption of an electric fleet would represent in a mine under Brazilian conditions, respecting the current regulatory standards.
\end{abstract}

Keywords: Mining ventilation; Electrical vehicles; Diesel; Heat emission.

\section{Introdução}

A ventilação em condições adequadas é fundamental para desenvolvimento das atividades em minas subterrâneas. Uma ventilação inadequada torna as condições ambientais da mina precárias para os operários e equipamentos representando para empresa uma perda de produtividade. A má qualidade do ar é uma das razões-chave porque ambientes subterrâneos podem ser perigosos para as pessoas que trabalham na mineração. Gases tóxicos como $\mathrm{CO}$ e $\mathrm{CO}_{2}$, outros gases inflamáveis e combustíveis e poeira estão, muitas vezes, naturalmente presentes. Por meio do fluxo de ar bem dimensionado, é possível controlar as concentrações de gases, de particulados e a temperatura em condições satisfatórias e seguras para a

'Programa de Pós-graduação em Engenharia Mineral - PPGEM, Departamento de Engenharia de Minas, Universidade de Ouro Preto - UFOP, Campus Morro do Cruzeiro, Ouro Preto, MG, Brasil.

*Autor correspondente: minas.leandro@gmail.com

2176-1523 C 2021. Costa et al. Publicado pela ABM. Este é um artigo publicado em acesso aberto (Open Access) sob a licença Creative Commons Attribution, que permite uso, distribuição e reprodução em qualquer meio, sem restrições desde que o trabalho original seja corretamente citado. 
operação da mina. $O$ consumo energético da ventilação pode chegar a $40 \%$ da energia empregada na mina [1].

Tecnologias inovadoras, como a ventilação por demanda (VOD), são aplicadas com sucesso em algumas minas subterrâneas apresentando resultados satisfatórios. Com o sistema VOD, a vazão de ar necessária é fornecida somente quando e onde necessário [2]. Entretanto, esta tecnologia não soluciona por completo dois desafios que a mineração subterrânea tende a enfrentar nas próximas décadas.

O primeiro desafio é o atendimento às legislações cada vez mais rigorosas quanto à exposição de trabalhadores aos particulados de diesel (DPM), com potencial cancerígeno. O segundo desafio está relacionado ao aprofundamento das minas, que resulta na necessidade de resfriamento intensivo para garantir temperaturas adequadas para a execução dos trabalhos com segurança [3].

Um dos impactos mais profundos na ventilação das minas subterrâneas nos últimos 100 anos foi a introdução de equipamentos a diesel. Além da emissão de poluentes, que podem comprometer a qualidade do ar e a saúde dos trabalhadores, esses equipamentos contribuem de forma significativa para o aumento da temperatura. A partir do momento que o equipamento a diesel começou a ser introduzido em minas canadenses na década de 1960, a frota tinha motores a diesel com potências menores que $75 \mathrm{~kW}$ (aproximadamente $100 \mathrm{HP}$ ), e eram poucos. Desde então, o número de equipamentos e a potência dos motores aumentaram significativamente, o que traz novos desafios e necessidade de ventilação mais eficiente [4].

Uma alternativa plausível para minimizar esse problema, porém que ainda representa certos desafios, é a adoção de frota $100 \%$ elétrica. Uma eletrificação total dos equipamentos implicará em zero emissão de gases e DPM; redução na emissão de calor, quando comparada à dos equipamentos a diesel e uma demanda por ventilação menor. Entretanto, a legislação para ventilação subterrânea ainda permanece inflexível e atrelada a parâmetros relacionados ao uso de diesel.

A evolução da tecnologia de baterias elétricas e as preocupações quanto ao preço do diesel já permitem a adoção de iniciativas isoladas de minas subterrâneas. Entretanto, informações sobre estes equipamentos e estudos técnicos de seu impacto na ventilação são escassos e difíceis de serem encontrados em domínio público, especialmente no Brasil [5].

\section{Materiais e métodos}

Para o desenvolvimento do artigo primeiramente foi realizado uma revisão bibliográfica abordando os impactos da frota a diesel no sistema de ventilação, técnicas de otimização da ventilação, impactos do excesso de calor na performance dos trabalhadores, vazões mínimas requeridas para equipamentos a diesel em alguns países e diferenças entre caminhões elétricos e a diesel. Depois é realizado o cálculo da economia gerada com a substituição total da frota diesel pela elétrica com auxílio de uma planilha eletrônica (excel) fundamentado em dados obtidos em catálogos dos fabricantes, sites especializados e artigos técnicos. Mas antes é preciso determinar a vazão necessária para o atendimento das normas.

No catálogo dos fabricantes é possível verificar informações sobre os equipamentos elétricos que mencionam que a velocidade de transporte é ligeiramente menor comparada aos modelos a diesel. Para carregadeiras LHD (que percorrem curtas distâncias), esse fato não se mostra muito relevante, mas para caminhões isso pode ser significativo. Por essa razão, será considerado potencial nominal de redução calculado termodinamicamente.

O segundo aspecto importante a ser analisado é se a redução da demanda de ventilação em função da implementação de frota elétrica (até o limite de 60\%) resultaria em uma ventilação deficiente. De acordo com Stinnette [6] e Mayrink [5] é sustentado que a demanda de ar para diluição de gases de desmonte $\left(0,028 \mathrm{~m}^{3} / \mathrm{s}\right.$ por $\left.\mathrm{kW}\right)$ é cerca de um terço daquela necessária para dissipação de calor $\left(0,075 \mathrm{~m}^{3} / \mathrm{s}\right.$ por $\mathrm{kW}$ para temperatura limite de $\left.20^{\circ} \mathrm{C}\right)$. Admitindo-se a redução da demanda por calor em $60 \%$, tem-se uma vazão de ar estimada de $0,03 \mathrm{~m}^{3} / \mathrm{s}$ por $\mathrm{kW}$. Trata-se de um valor muito próximo ao necessário para dissipação de gases de desmonte. Portanto, nessa redução não há margem segura para dispersar corretamente os gases do desmonte. Será adotada uma redução de $40 \%$, pois com uma vazão de $0,045 \mathrm{~m}^{3} / \mathrm{s}$ por $\mathrm{kW}$ os autores consideram suficientemente seguro para garantir a dissipação de gases de desmonte $\left(0,028 \mathrm{~m}^{3} / \mathrm{s}\right.$ por $\left.\mathrm{kW}\right)$.

Entretanto, outro aspecto a ser considerado sobre o desmonte é o potencial impacto que a redução da velocidade de ar poderia trazer ao tempo de limpeza/carreamento de gases da frente de lavra. Uma redução da vazão em $40 \%$ assegura satisfatoriamente a segurança (gases de desmonte) e o baixo impacto no ciclo operacional. Considerando o múltiplo regulatório mais comumente empregado no mundo para vazão de ar regido por equipamentos a diesel $\left(0,06 \mathrm{~m}^{3} / \mathrm{s}\right.$ por $\left.\mathrm{kW}\right)$, esta redução representaria um múltiplo de $0,036 \mathrm{~m}^{3} / \mathrm{s}$ por $\mathrm{kW}$, constituindo uma infração à norma. No caso da legislação brasileira [7], é $0,08 \mathrm{~m}^{3}$ por $\mathrm{kW}$, o novo valor seria de $0,048 \mathrm{~m}^{3} / \mathrm{s}$ por $\mathrm{kW}$, também, configurando uma infração. Esses dois casos reafirmam a necessidade de revisão da norma adaptada a esta nova tecnologia.

\section{Revisão bibliográfica}

O acesso a depósitos minerais está ficando mais difícil nas minas subterrâneas. Os níveis de lavra estão aprofundando cada vez mais exigindo mais caminhões e mais tempo de transporte devido ao aumento das distâncias. Quase todas as minas subterrâneas no mundo utilizam equipamentos movidos a diesel. A organização mundial de saúde (OMS) menciona que o material particulado do diesel (DPM) é cancerígeno e a exposição a elevadas concentrações de DPM causa efeitos 
nocivos à saúde. Além disso, os equipamentos movidos a diesel correspondem a $30 \%$ das fatalidades ocorridas na mineração em 2018 [8].

Para auxiliar na melhoria das condições ambientais, controle e no gerenciamento da quantidade de vazão de ar necessária em uma mina pode-se aplicar a ventilação por demanda (VOD) e os conhecimentos sobre a aerodinâmica dos ventiladores. O conceito de VOD não é novo para mineração, mas foi experimentado e testado para diferentes níveis de sucesso ou fracasso em todo o mundo. Diversos pesquisadores relataram as experiências obtidas em países diferentes [9].

A quantidade de ar fresco necessária por equipamento a diesel foi discutida, pela primeira vez, em minas da Suécia em 1979 [10]. Hatakeyama et al. [11], por meio de vários anemômetros espalhados na mina, monitorou e controlou a concentração de monóxido de carbono e gás metano em minas de carvão no Japão. No entanto, somente nos últimos 10 anos as condições econômicas e a evolução tecnológica de equipamentos e softwares favoreceram a implementação em diferentes níveis na América do Norte, América do Sul e Norte da Europa [9]. Os estudos tinham como objetivo reduzir significativamente o consumo de energia sem negligenciar aspectos da quantidade e qualidade de ar necessários para o melhor desempenho do sistema, atendendo às normas de segurança e conforto dos trabalhadores, impostas pelos órgãos de fisscalização.

A transferência de calor não é governada por uma única relação, mas por uma combinação de várias leis da Física. Em muitas situações práticas, a transferência de calor de uma superfície ocorre simultaneamente por convecção (para o ar ambiente) e por radiação (para a vizinhança).

A energia da radiação emitida por um corpo é transmitida no espaço. Somente no vácuo a radiação se propaga sem nenhuma atenuação. $\mathrm{O}$ ar atmosférico de um ambiente, como uma sala, por exemplo, tem atenuação da radiação pelo ar considerada insignificante.

Patel e Patel [12] investigaram diferentes parâmetros que afetam o desempenho do ventilador axial em minas subterrâneas. Esses são o ângulo de inclinação, o número de lâminas, ângulo da lâmina, erosão e corrosão das lâminas, redução do espaço de ar em um circuito fechado, entre outros. Alterando o ângulo de inclinação da lâmina para $44^{\circ}, 54^{\circ}, 59^{\circ}, 64^{\circ}$, respectivamente, o valor do fluxo de ar é $1175 \mathrm{~m}^{3} / \mathrm{h}, 1270 \mathrm{~m}^{3} / \mathrm{h}, 1340 \mathrm{~m}^{3} / \mathrm{h}$ e $800 \mathrm{~m}^{3} / \mathrm{h}$.

Assim, para aumentar a taxa de fluxo, o ângulo de inclinação da lâmina do ventilador deve ser mantido em torno de $59^{\circ}$. O aumento do número de pás de um para dois resulta em um aumento de $6 \%$ na eficiência aerodinâmica. Mais uma vez, reduzindo o espaço de ar entre a ponta do rotor e o revestimento, reduzem-se as perdas de ar e melhora-se o desempenho do ventilador.

A maioria dos sistemas de ventilação tem uma baixa eficiência (65\%) [1]. O autor propõe 3 estratégias para a redução de custos para o funcionamento eficiente da ventilação. A primeira seria ampliação da abertura de saída do ar dos cones. Essa medida reduziria as perdas de pressão gerando uma economia anual de potência de $38 \mathrm{~kW}(18 \%)$. Seriam necessários investimentos de US\$ 60.000 para a construção e instalação de novos cones e o retorno dos investimentos seria de 2 anos devido a economia de energia. A segunda medida, sem incorrer em custos de investimento, seria uma mudança na operação do ventilador para uma configuração de meia-lâmina com inclinação de $22^{\circ}$; essas condições forneceriam o mesmo fluxo necessário, mas com um aumento da eficiência de $59,5 \%$. A potência necessária seria reduzida para $85,9 \mathrm{~kW}$ e o custo operacional anual seria reduzido em $22 \%$. A última estratégia seria a instalação e manutenção correta do duto que poderia reduzir os custos operacionais do ventilador auxiliar em $20 \%$ ou mais.

Detalham-se a seguir os impactos na temperatura, umidade e nos custos da substituição da frota movida a diesel pela frota elétrica.

\subsection{Controle de calor e temperatura}

O controle da temperatura e da umidade é devido à necessidade de se mantê-las em faixas adequadas para execução dos trabalhos de maneira segurança e eficiente no ambiente subterrâneo [13].

Um bom padrão a ser seguido é manter a temperatura efetiva entre $21^{\circ} \mathrm{Ce} 26,5^{\circ} \mathrm{C}$, pois nesse intervalo o conforto humano é satisfatório e o rendimento de seu trabalho é máximo [14]. Quando a temperatura estiver entre $26,5^{\circ} \mathrm{Ce}$ $30^{\circ} \mathrm{C}$ recomenda-se resfriamento do ambiente para assegurar melhor conforto. Abaixo dos $16^{\circ} \mathrm{C}$ a sensação de frio torna o ambiente desconfortável. Entretanto entre $16^{\circ}$ e $21^{\circ} \mathrm{C}$ não se verifica ganho aparente em desempenho que justifique resfriamento. A Figura 1 mostra a relação entre temperatura e desempenho do trabalho humano.

No dimensionamento de sistemas de ventilação determinar vazão de ar necessária $\left(\mathrm{m}^{3} / \mathrm{s}\right.$ ou $\left.\mathrm{m}^{3} / \mathrm{min}\right)$ significa assegurar um ambiente seguro à operação sendo um parâmetro chave de projeto e que norteia a legislação mineral [5]. A Tabela 1 apresenta as vazões mínimas exigidas pela legislação de alguns países mineradores.

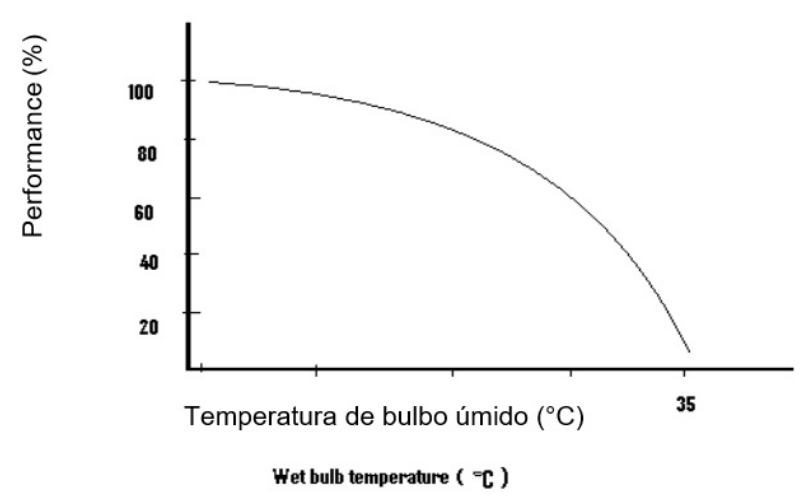

Figura 1. Performance dos trabalhadores em relação à temperatura de bulbo úmido [13]. 
Tabela 1. Legislações mundiais sobre demanda por ventilação para equipamentos a diesel [6]

\begin{tabular}{|c|c|}
\hline País & Legislação sobre demanda de ventilação \\
\hline \multirow{2}{*}{ Austrália } & $0,06 \mathrm{~m}^{3} / \mathrm{s}$ por $\mathrm{kW}$ (mínimo; New South Wales) \\
\hline & 0,05 m³/s por kW (mínimo; Western Austrália) \\
\hline Canadá & $\begin{array}{l}\text { Específico para cada província, entre } 0,045-0,092 \\
\mathrm{~m}^{3} / \mathrm{s} \text { por } \mathrm{kW} \text { (mínimo). Usualmente: } 0,06 \mathrm{~m}^{3} / \mathrm{s} \text { por } \mathrm{kW}\end{array}$ \\
\hline Chile & $\begin{array}{l}2,83 \mathrm{~m}^{3} / \mathrm{min} \text { por HP (mínimo) } \\
\text { (Equivalente a } 0,063 \mathrm{~m}^{3} / \mathrm{s} \text { por } \mathrm{kW} \text { ) }\end{array}$ \\
\hline China & $0,067 \mathrm{~m}^{3} / \mathrm{s}$ por $\mathrm{kW}$ \\
\hline África do Sul & $0,063 \mathrm{~m}^{3} / \mathrm{s}$ por $\mathrm{kW}$ (mínimo baseado em "boas práticas") \\
\hline Indonésia & $0,067 \mathrm{~m}^{3} / \mathrm{s}$ por $\mathrm{kW}$ \\
\hline Brasil & $3,5 \mathrm{~m}^{3} / \mathrm{min}$ por $\mathrm{HP}$ - (Equivalente a $0,08 \mathrm{~m}^{3} / \mathrm{s}$ por $\mathrm{kW}$ ) \\
\hline
\end{tabular}

Kerai e Halim [15] e Stinnette [6] sustentam que a determinação do parâmetro de vazão em projetos de mineração subterrânea é um processo complexo, porém essencial para o sucesso do empreendimento. Se superdimensionado, implica custos de investimento e operacionais que podem comprometer em algum nível a economicidade do empreendimento; mas se subdimensionados poderá implicar restrições operacionais (ritmo/escala de produção) e riscos aos trabalhadores que podem levar à interdição da operação.

Sabe-se também que os equipamentos não trabalham a plena carga, a todo tempo. Para modelar com precisão a quantidade de calor gerado por máquinas acionadas a diesel, é essencial usar a taxa de utilização desses equipamentos, preferencialmente pela medição de seu ciclo operacional, em uma média mensal. Pode-se também estimar a carga térmica com mais precisão, quando se sabe o consumo de diesel, multiplicado por sua capacidade calorífica. Essas cargas caloríficas podem ser localizadas ou distribuídas [13].

Em um cenário, em que as empresas estão cada vez mais pressionadas no atendimento às normas ambientais e de segurança, a utilização dos equipamentos elétricos representa uma alternativa no cumprimento da legislação. Muitas mineradoras questionam a respeito do rigor da norma e na dificuldade a seu atendimento [16].

Estudos apontam potencial redução na demanda de ar para dissipação de calor em até $40 \%$ com a frota elétrica [6]. Como a legislação baseia-se em um parâmetro de potência instalada a diesel, em um cenário de eletrificação dos equipamentos não há precedente regulatório que norteie cientificamente o cálculo para esta modalidade.

\subsection{Diferenças entre equipamentos a diesel e elétricos}

Os equipamentos a diesel introduziram novos riscos relacionados com os subprodutos de sua combustão em ambientes confinados, que podem tornar irrespirável o ar no interior das minas ou mesmo danosa à saúde dos trabalhadores [17].

Dependendo da escala de produção da mina, equipamentos a diesel podem estar presentes em maior ou menor quantidade, porém contribuem para a emissão de gases tóxicos $\left(\mathrm{CO}, \mathrm{CO}_{2}, \mathrm{NO}_{x}, \mathrm{H}_{2} \mathrm{~S}\right)$, particulado de diesel
(DPM) e emissão de calor (via exaustão de gases e perdas térmicas do motor).

Para o emprego do diesel, inicialmente é utilizada a quantidade estipulada, de $0,08 \mathrm{~m}^{3} / \mathrm{s}$ por $\mathrm{kW}$ e depois, com o advento do combustível diesel S10, a NR 22/1978 estipulou $0,06 \mathrm{~m}^{3} / \mathrm{s}$, ou, no Canadá, 0,032 a $0,063 \mathrm{~m}^{3} / \mathrm{s}$ por $\mathrm{kW}$. Nesse país, a Vale Inco prefere trabalhar com $0,079 \mathrm{~m}^{3} / \mathrm{s}$ por $\mathrm{kW}$. Na Mina Ballarat, a estimativa considerou $0,05 \mathrm{~m}^{3} / \mathrm{s}$ por $\mathrm{kW}$ [13].

Devido à eficiência geral dos motores de combustão interna, o equipamento a diesel pode produzir aproximadamente três vezes mais calor que o equipamento elétrico [13]. Diante desse contexto, os fabricantes de equipamentos para mineração subterrânea buscaram desenvolver alternativas para substituição do diesel como, por exemplo, equipamentos movidos a energia elétrica.

Com o aprofundamento das minas as mineradoras enfrentam problemas com a elevada temperatura e baixa concentração de oxigênio que exigem ventilação intensa e até mesmo resfriamento do ar. Nesse cenário, a operação de equipamentos a diesel torna-se um agravante pelo calor e gases/particulados emitidos. Além do calor gerado e de uma certa quantidade de água e $\mathrm{CO}_{2}$, a combustão produz outros gases e poluentes que afetam o ambiente de trabalho e aumentam custos de ventilação da mina.

A mudança para caminhão elétrico elimina esse custo. É mais barato carregar uma bateria do que operar um ventilador. A grande dificuldade da implantação desse equipamento é o elevado investimento inicial, sendo 15 a $55 \%$ mais caro do que os equipamentos a diesel [18]. Há possível potencial de redução da demanda de ventilação por calor da ordem de $80 \%$, ou seja, elétricos emitiriam cerca de $20 \%$ do calor de um diesel. Entretanto, estes números são baseados na capacidade nominal de carga dos equipamentos (toneladas) e não incluem fatores operacionais tais como tempo de ciclo, velocidade de transporte, load factor, entre outros que podem impactar no número de equipamentos necessários para atingir a produção desejada [19].

Levantamentos feitos por Moore [20] e Paraszczak et al. [21] citados por Mayrink [5] mencionam operações de caminhões elétricos em minas na Suécia, Canadá, Austrália, Espanha, EUA e Kazaquistão com diferentes níveis de sucesso. Ressaltam-se apenas quatro operações que utilizaram com sucesso e em escala operacional caminhões elétricos em subterrâneo: as minas Coleman-McCreedy (Canadá), Creighton (Canadá) e Stillwater (EUA) operaram caminhões elétricos do modelo Kiruna; enquanto a mina Kidd Creek (Canadá) operou caminhão elétrico modelo trolley INCO. Entretanto, pode-se dizer que a partir de meados de 2010 a temática de adoção de frota elétrica em larga escala em mina subterrânea voltou a ganhar destaque em publicações e estudos técnicos e corporativos, tais como Jacobs [23], McCrae [22], Kerai e Halim [15] e Varaschin [19].

Leonida [8] menciona outras opções viáveis como a utilização de células de combustível de hidrogênio e equipamentos movidos a diesel com tecnologias que 
minimizem a emissão de gases; melhores tecnologias de baterias com estação de recarga que pode ser operada por controle remoto. Sistema móvel fornece recarga para 3-4 h de trabalho. Outro sistema permite recarga da bateria fora do equipamento.

\section{Estudo de caso}

O objetivo do estudo de caso é avaliar o potencial impacto que a implementação da frota elétrica representaria em uma mineradora de ouro, já em produção, localizada no estado de Minas Gerais. As Tabelas 2 e 3 apresentam, respectivamente, dados de produção e ventiladores principais utilizados.

Analisando-se a frota de equipamentos da Mina A, verifica-se que a quase totalidade dos equipamentos é movida a diesel (exceto alguns casos de perfuratrizes jumbos/fandrill - que apresentam alguma unidade motriz elétrica). A mudança das carregadeiras e caminhões por elétricos reduziu o calor sensível e latente em $56 \%$ e $73 \%$, respectivamente [24].

No trabalho realizado por Massanés et al. [25] a troca de carregadeiras caminhões a diesel por elétricos reduziu de $74 \%$ o calor vindo das máquinas para $52 \%$.

$\mathrm{Na}$ Mina Creighton medidas de temperatura em 5 locais, no manuseio de minério, em alguns turnos, tiveram variação de 29 a $35,5^{\circ} \mathrm{C}$ [26]. Autores concluíram, após avaliação de tempos e movimentos, que a ventilação auxiliar é necessária continuamente apenas entre chegada do veículo de carregamento, sua atividade e até 10 min após a última partida. Em estudos feitos na mina Jinqu Gold (localizada na China) após otimização, reduziu-se $5-6^{\circ} \mathrm{C}$, com resfriamento [27].

Tabela 2. Dados gerais sobre escala de produção da Mina A

\begin{tabular}{cc}
\hline \multicolumn{3}{c}{ MINA A } \\
\hline Localização & Minas Gerais - Brasil \\
Produção & 642.865 tpa (Minério) \\
& $140 \mathrm{kOz} /$ ano \\
Dias trabalhados & 341 \\
$\mathrm{~N}^{\circ}$ de turnos & 4 \\
Horas trabalhadas/turno & 4,8 \\
Horas programadas/ano & 6547 \\
\hline
\end{tabular}

Para buscar-se avaliar os potenciais impactos econômicos na ventilação, é realizada uma análise com base na demanda de ventilação estimada $\left(\mathrm{m}^{3} / \mathrm{s}\right)$. A metodologia mais correta seria redimensionar a potência instalada dos ventiladores (em $\mathrm{kW}$, principais e secundários) para a nova demanda de ventilação. Isso é possível utilizando a ventilação por demanda (VOD) por meio de inversores de frequência para modular a velocidade dos ventiladores. A Tabela 4 demonstra um comparativo de cenários entre aplicação da frota elétrica e a diesel.

O custo médio operacional (OPEX) de um sistema de ventilação é da ordem de US\$ 12.185,00/ano por m³/s (admitindo-se uma operação anual do sistema sem interrupções, 365 dias $\times 24$ horas) [28]. Os custos de energia associados aos sistemas de ventilação operacional são o segundo maior custo de produção da mina após a mão de obra [1]. O custo da ventilação é de cerca de US\$35,71 por metro cúbico $\left(\mathrm{m}^{3}\right)$. Cada cavalo-vapor do motor diesel exige cerca de $4,2 \mathrm{~m}^{3}$ por minuto de ar. Dependendo do custo da eletricidade em uma determinada mina, isso pode custar cerca de US\$150.000,00 em média por máquina, apenas nos custos de ventilação [33].

Uma outra opção sugerida por Lafuente [34] seria a aplicação dos equipamentos híbridos. O sistema de acionamento apresenta um motor diesel e quatro motores elétricos. Comparado com o equipamento a diesel a demanda de ar é 33\% menor. Os custos totais (operacional, manutenção, combustível e aquisição) são 29\% menores [10].

Nos estudos na Borden Mine (localizada no Canadá), das questões avaliadas pelos operadores, $79 \%$ consideraram o desempenho do equipamento elétrico superior (ou muito superior) ao movido a diesel e $98 \%$ consideraram grande (ou muito grande) a melhoria da qualidade do ar [35].

Segundo estudos feitos na mina de ouro, Macassa (Canadá), houve um aumento de apenas $2{ }^{\circ} \mathrm{C}$ na frente de lavra em operação $100 \%$ elétrica contra $8^{\circ} \mathrm{C}$ em operação à diesel [36].

Gleeson [36] menciona que na mina Kittilä Gold (localizada no norte da Finlândia), os operadores observaram menos geração de calor, vibrações e melhor qualidade do ar no ambiente operacional. A empresa havia decidido usar equipamentos elétricos a bateria há cinco anos, devido ao aprofundamento da mina. A expectativa é atingir $1.040 \mathrm{~m}$ de profundidade como parte de um plano de expansão para aumentar a produção em $25 \%$ para $2 \mathrm{Mt} /$ ano de minério.

Tabela 3. Capacidade nominal dos sistemas de ventilação da Mina A

\begin{tabular}{|c|c|c|c|c|c|c|}
\hline \multicolumn{7}{|c|}{ MINA A } \\
\hline \multicolumn{7}{|c|}{ VENTILADORES PRINCIPAIS - CAPACIDADE NOMINAL INSTALADA } \\
\hline \multirow[b]{2}{*}{ Modelo } & \multirow{2}{*}{$\begin{array}{l}\text { Número de } \\
\text { Ventiladores }\end{array}$} & \multicolumn{4}{|c|}{ Dados Unitários dos Ventiladores } & \multirow[b]{2}{*}{$\begin{array}{c}\text { Vazão Total } \\
\qquad\left(\mathbf{m}^{3} / \mathbf{s}\right)\end{array}$} \\
\hline & & Potência (CV) & $\begin{array}{c}\text { Vazão de Ar } \\
\left(\mathrm{m}^{3} / \mathrm{s}\right)\end{array}$ & Pressão (kPa) & Rotação (rpm) & \\
\hline AXIAL & 2 & 125 & 40 & 3,06 & 1780 & 270 \\
\hline AXIAL & 2 & 500 & 70 & 3,4 & 1785 & 399 \\
\hline \multirow[t]{2}{*}{ CENTRÍFUGO } & 2 & 500 & 115 & 2,5 & 590 & 266 \\
\hline & & \multicolumn{4}{|c|}{ VAZÃO TOTAL INSTALADA $\left(\mathrm{m}^{3} / \mathrm{s}\right)$} & 460 \\
\hline
\end{tabular}


Tabela 4. Comparativo de cenários da Mina A com adoção de frota elétrica

\begin{tabular}{|c|c|c|}
\hline & $100 \%$ Diesel & $100 \%$ Elétrica \\
\hline \multicolumn{3}{|l|}{ Ventilação } \\
\hline Vazão requerida $\left(\mathrm{m}^{3} / \mathrm{s}\right)$ & 460 & 276 \\
\hline Custo da Ventilação Total $\left(10^{6} \$ / \text { ano }\right)^{(1)}$ & 47,23 & 30 \\
\hline \multicolumn{3}{|l|}{ Combustível } \\
\hline Diesel (ML) & 8,5 & 0,0 \\
\hline LHD - $(30 \mathrm{~L} / \mathrm{h})^{(2)}$ & 2,4 & - \\
\hline Caminhão - $(45 \mathrm{~L} / \mathrm{h})^{(2)}$ & 3,8 & - \\
\hline Auxiliar - $(14 \mathrm{~L} / \mathrm{h})^{(3)}$ & 2,3 & - \\
\hline Energia elétrica (GWh) ${ }^{(4)}$ & 0,0 & 44,6 \\
\hline LHD - (70\% Nominal Diesel) & - & 11,5 \\
\hline Caminhão - (70\% Nominal Diesel) & - & 20,6 \\
\hline Auxiliar - (70\% Nominal Diesel) & - & 12,5 \\
\hline Combustível Total ( $10^{6} \$ /$ ano $)$ & 33,37 & 18,51 \\
\hline $\operatorname{Diesel}(\$ 0,95 / \mathrm{L})^{(5)}$ & $\mathrm{R} \$ 33,37$ & - \\
\hline Eletricidade $(\$ 0,1 / \mathrm{kWh})^{(6)}$ & - & $\mathrm{R} \$ 18,51$ \\
\hline \multicolumn{3}{|l|}{ OPEX (M\$/ano) } \\
\hline \multirow[t]{2}{*}{ Excluso Manutenção } & & 32,47 \\
\hline & & $-57 \%$ \\
\hline \multirow[t]{2}{*}{ Emissão $\mathrm{CO}_{2}(\mathrm{Kt})^{(7)}$} & 22,6 & 0 \\
\hline & & $-100 \%$ \\
\hline
\end{tabular}

Foi necessário adaptar a mina com a infraestrutura necessária de carregamento/troca de bateria.

Um estudo comparativo entre a frota utilizada a diesel $(100 \%)$ e a frota elétrica $(100 \%)$ foi realizado na Mina A. Observa-se na Tabela 4 uma redução significativa na vazão requerida e nos custos da ventilação. É importante destacar a diminuição da geração de calor e de gases tóxicos contribuindo para um ambiente mais seguro e saudável.

\section{Conclusões}

Aplicação de equipamentos elétricos em minas subterrâneas já se apresenta como uma realidade no setor em alguns países, como Estados Unidos, Canadá e Suécia. Diante do alto risco inerente do setor mineral, a pressão por resultados financeiros, fiscalização rígida dos órgãos trabalhistas e ambientais a aplicação desta tecnologia em substituição ao diesel desponta como promissora.
Aplicação dessa modalidade proporciona a redução de emissões de gases estufa (ex: $\mathrm{CO}_{2}$ ), redução significativa do consumo de energia elétrica, da demanda de ventilação devido à menor emissão de calor e zero exposição aos particulados diesel. Porém, devido ao custo de aquisição e o predomínio dos combustíveis fosseis (petróleo), a utilização da energia elétrica em equipamentos móveis encontra verdadeiras barreiras para sua utilização.

Os resultados obtidos com o estudo de caso de uma mina no Brasil, ainda que em uma análise preliminar, demonstraram o interesse econômico da implementação de frota totalmente elétrica em subterrâneo no cenário nacional.

Para uma redução em 40\% da demanda de ventilação, pode-se verificar uma redução potencial de até $40 \%$ em custos operacionais e redução de até $100 \%$ na emissão de $\mathrm{CO}_{2}$, aparentemente sem prejuízos às condições de saúde. Além da significativa redução de custos, verifica-se o potencial benefício ambiental com a redução na emissão de gases e melhora na qualidade da saúde.

\section{Referências}

1 Souza E. Cost saving strategies in mine ventilation. Ontario, Canada: Buchan Department of Mining, Queen's University Kingston; 2015.

2 Tran-Valade T, Allen C. Ventilation-on-demand consideration for the business case. In: Proceedings of the Toronto CIM Conference; 2013; Toronto. Westmount, QC: CIM; 2013.

3 Xiaojie Y, Qiaoyun H, Jiewen P, Xiaowei S, Dinggui H. Chao 1. Progress of heat hazard treatment in deep mines. Mining Science and Technology. 2011;21(2):295-299. 
4 Kocsis C, Hardcastle S, Keen B. A heat study and the modelling of future climatic conditions at Vale Inco's Coleman Mc Creedy East Mine. In: Proceedings of the 12th U.S. North American Mine Ventilation Symposium; 2008; Reno, NV, USA. Reno, NV: University of Nevada; 2008. p. 203-210.

5 Mayrink AG. Avaliação do impacto na demanda de ventilação em mina subterrânea com a adoção de frota elétrica [monografia]. Belo Horizonte: Escola de Engenharia, Universidade Federal de Minas Gerais; 2019.

6 Stinnette JD. Establishing total airflow requirements for underground metal/nonmetal mines based on the diesel equipment fleet [thesis]. Kingston: Queen's University; 2013.

7 Brasil. Ministério do Trabalho e Emprego. NR 22: segurança e saúde ocupacional na mineração. Brasília; 2019 [acesso em 20 out. 2019]. Disponível em: http://www.trtsp.jus.br/geral/tribunal2/LEGIS/CLT/NRs/NR_22. html.1978

8 Leonida C. Battery electric vehicles: brightening the mining industry's future. Engineering Mining Journal. 2020 [acesso em 18 abr. 2020];(jan.):32-33. Disponível em: https://www.e-mj.com/flipbooks/january-2020.

9 Acuña EI, Allen C. Ventilation Control System implementation and energy consumption reduction at Totten Mine with Level 4 'Tagging' and future plans. In: Proceedings of the 1st International Conference on Underground Mining Technology; 2017; Sudbury, Canada. Australia: Australian Centre for Geomechanics; 2017.

10 Rustan A. Review of developments in monitoring and control of mine ventilation systems. In: Proceedings of the 2nd International Mine Ventilation Congress; 1979; Reno, NV. New York: Society of Mining Engineers of American Institute of Mining, Metallurgical, and Petroleum Engineers; 1979. p. 223-229.

11 Hatakeyama Y, Sakai T, Kimura Y, Inoue M. Real time ventilation network analysis based on anemometers in an underground coal mine. In: Proceedings of the 5th International Mine Ventilation Congress; 1992; Johannesburg. Johannesburg: Mine Ventilation Society of South Africa; 1992. p. 345-350.

12 Patel JS, Patel SM. Parameter affecting the performance of axial fan performance. International Journal of Engineering Research \& Technology. 2012;1(3):1-3.

13 McPherson MJ. Subsurface ventilation engineering. 1st ed. Londres: Chapman \& Hall; 1993. 905 p.

14 Johnson B. Heat, humidity and air conditioning. In: Hartman HL, editor. SME mining engineering handbook. 2nd ed. Vol. 1. Colorado: Society for Mining, Metallurgy and Exploration; 1992. Cap. 11.4, p. 1028-1039.

15 Kerai M, Halim A. Ventilation requirement for electric vehicles in underground hard rock mines: a conceptual study. In: Proceedings of the Australia Mine Ventilation Conference; 2013; Adelaide. Carlton: The Australasian Institute of Mining and Metallurgy; 2013.

16 Martins C. Avaliação das normas reguladoras de mineração para minas subterrâneas no brasil e da legislação mineral brasileira para segurança em subsolo [dissertação]. Ouro Preto: Universidade Federal de Ouro Preto; 2016.

17 Brake DJ, Nixon T. Correctly estimating primary airflow requirements for underground metalliferous mines. In: Proceedings of the 10th Underground Operator's Conference; 2006; Launceston. Australia: AusIMM; 2006.

18 Ross I. Goldcorp going electric with Chapleau gold mine. Northern Ontario Business. 2016 [acesso em 8 jun. 2019]. Disponível em: https://www.northernontariobusiness.com/industry-news/mining/

19 Varaschin J. The economic case for electric mining equipment and technical considerations relating to their implementation [dissertation]. Kingston: Queen's University; 2016.

20 Moore P. Plugging the gap underground. Mining Magazine. 2010;(nov.):40-46.

21 Paraszczak J, Svedlund E, Fytas K, Laflamme M. Electrification of loaders and trucks - a step towards more sustainable underground mining. Renewable Energy and Power Quality Journal. 2014;12(12):81-86.

22 McCrae MA. How batteries are lowering the costs of underground mining. Mining DOTcom. 2012 Maio 12 [acesso em 8 jun. 2019]. Disponível em: https://www.mining.com/web/battery-technology-is-moving-incredibly-fast/

23 Jacobs W. Electric LHDs in underground hard rock mining: a cost/benefit analysis [monografia]. Australia: University of Western Austrália; 2013. 62 p.

24 Costa LV. Análise via simulação da ventilação em mina subterrânea: estudo de caso mina córrego do Sítio I [tese]. Ouro Preto: Programa de Pós-graduação em Engenharia Mineral, Universidade Federal de Ouro Preto; 2019.

25 Massanés MB, Castañón A, Sanmiquel L. Heat flow assessment in an underground mine: an approach to improve the environmental conditions. Dyna. 2016;83(197):174-179.

26 Hardcastle S, Kocsis C, O' Connor D. Justifying ventilation on demand in a Canadian mine and the need for process based simulations. In: Proceedings of the 11th U.S. North American Mine Ventilation Symposium; 2006; Pennsylvania, USA. London: Taylor \& Francis; 2006. p. 15-21. 
27 Nie X, Wei X, Li X, Lu C. Heat treatment and ventilation optimization in a deep mine. Advances in Civil Engineering. 2018;2018(4):1-12.

28 ABB Inc. Underground electric haulage trucks: introduction and benefits. In: Proceedings of the XVI Annual MDEC Conference; 2010 July; Toronto, Canada. Toronto: MDEC; 2011 [acesso em 16 nov. 2019]. Disponível em: http:// mdec.ca/2010/S3P3_willick.pdf

29 Caterpillar Inc. Caterpillar performance handbook. 41st ed. Illinois: Caterpillar Inc., 2011. Estimating owning and operating costs; cap. 20, 1285 p. [acesso em 8 ago. 2019]. Disponível em: https://www.holtcat.com/Documents/ PDFs/2012PerformanceHandbook/

30 Global Petrol Prices. Brasil: preços do gasóleo. 2019 [acesso em 27 mar. 2020]. Disponível em: https:// pt.globalpetrolprices.com/Brazil/diesel_prices

31 Firjan. Quanto custa a energia elétrica para a indústria no Brasil? Rio de Janeiro: Firjan; 2017 [acesso em 8 dez. 2019]. Disponível em: https://www.firjan.com.br/publicacoes/publicacoes-de-economia/

32 U.S. Environmental Protection Agency. EPA420-F-05-001: emission facts: average carbon dioxide emissions resulting from gasoline and diesel fuel. Washington: EPA; 2005 [acesso em 16 set. 2019]. Disponível em: https:// nepis.epa.gov/Exe

33 Cholewa P. Why the mines of the future will be diesel free. 2017 [acesso em 29 mar. 2020]. Disponível em: https:// medatech.ca/diesel-free-mining

34 Lafuente GER. Introduccion de LHD hibrido a la industria minera [dissertação]. Santiago: Universidad de Chile; 2017 [acesso em 29 mar. 2020]. Disponível em: http://repositorio.uchile.cl

35 Calnan P, Young JY. Update on electric U/G equipment. Ontario: Mine Borden Gold; 2017.

36 Gleeson D. Epiroc and Kirkland Lake Gold leading the mine electrification race. The International Mining. 2018 Nov 14 [acesso em 27 mar. 2020]. Disponível em: https://im-mining.com/2018/11/14/epiroc- kirkland-lake-goldleading-mine-electrification-race-riach-says/

Recebido em: 11 Jun. 2020

Aceito em: 26 Jan. 2021 